\title{
$11,14,19$
}

\section{О формуле Леонтьева для параметра Грюнайзена твердых тел}

\author{
(C) Д.С. Сандитов ${ }^{1,2}$, А.А. Машанов ${ }^{1}$ \\ ${ }^{1}$ Бурятский государственный университет им. Доржи Банзарова, \\ Улан-Удэ, Россия \\ ${ }^{2}$ Институт фризического материаловедения СО РАН, \\ Улан-Удэ, Россия \\ E-mail: Sanditov@bsu.ru
}

Поступила в Редакцию 21 сентября 2020 г.

В окончательной редакции 29 сентября 2020 г.

Принята к публикации 30 сентября 2020 г.

\begin{abstract}
Установлено, что формула Леонтьева находится в удовлетворительном согласии с уравнением Грюнайзена. Дается комментарий к выводу данной формулы. Обсуждается кажущееся противоречие в ней. Рассмотрено ее применение к кристаллическим и стеклообразным твердым телам. Показано, что из формулы Леонтьева можно вывести соотношение Беломестных-Теслевой, согласно которому параметр Грюнайзена оказывается однозначной функцией коэффициента Пуассона.
\end{abstract}

Ключевые слова: упругие и тепловые свойства, ангармонизм, уравнение Грюнайзена, скорости акустических волн, формулы Леонтьева и Беломестных-Теслевой, кристаллы, стекла.

DOI: 10.21883/FTT.2021.02.50482.202

\section{1. Введение}

В уравнение состояния твердых тел входит параметр Грюнайзена, характеризующий нелинейность силы межатомного взаимодействия и ангармонизм колебаний решетки. Он определяется изменением частоты нормальных мод колебаний решетки $v_{i}$ в зависимости от изменения объема

$$
\gamma_{i}=-\frac{V}{v_{i}}\left(\frac{d v_{i}}{d V}\right)=-\frac{d \ln v_{i}}{d \ln V}
$$

где $\gamma_{i}$ иногда называют частотным параметром Грюнайзена. В большинстве случаев используется приближение, в котором все нормальные моды колебаний имеют одинаковую зависимость частоты от объема

$$
\gamma=-\frac{d \ln v}{d \ln V}
$$

Мы следуем данному приближению и не пользуемся соотношением (1). Обычно $\gamma$ вычисляется по уравнению Грюнайзена (2) из экспериментальных данных о коэффициенте объемного теплового расширения $\beta$, изотермическом модуле объемного сжатия $B$, молярном объеме $V$ и молярной теплоемкости $C_{V}$

$$
\gamma=\frac{\beta B V}{C_{V}},
$$

которое выводится из уравнения состояния. Здесь $\gamma$ называют термодинамическим параметром Грюнайзена. В настоящей работе рассматривается именно этот термодинамический параметр, который часто называют просто параметром Грюнайзена.
Леонтьев [1] выполнил усреднение частоты нормальных мод колебаний решетки $v_{i}$ и непосредственно из определения $\gamma_{i}(1)$ получил следующее уравнение для параметра Грюнайзена

$$
\gamma=\frac{3}{2}\left(\frac{B_{A}}{\rho v_{K}^{2}}\right),
$$

где $B_{A}-$ адиабатический модуль объемного сжатия, $\rho$ - плотность, $v_{K}-$ среднеквадратичная скорость звука

$$
v_{K}^{2}=\frac{v_{L}^{2}+2 v_{S}^{2}}{3},
$$

$v_{L}$ и $v_{S}-$ скорости продольной и поперечной акустических волн, соответственно. Формула Леонтьева привлекательна тем, что в отличие от уравнения Грюнайзена она позволяет рассчитывать $\gamma$ по более доступным экспериментальным данным. Однако ни самим автором, ни другими исследователями эта формула не сравнивалась с уравнением Грюнайзена, она была использована для других целей. Считаем, что формула Леонтьева несомненно заслуживает внимания.

Настоящая работа посвящена непосредственному сопоставлению уравнений Леонтьева и Грюнайзена, а также обоснованию оправданности применения соотношения для давления газа при выводе формулы Леонтьева. Обсуждается ее особенность, связанная с ангармонизмом. Предлагается расчет $\gamma$ по этой формуле для кристаллов и стеклообразных систем. Рассмотрена связь уравнения Леонтьева с формулой Беломестных-Теслевой [2]. 


\section{2. Сравнение соотношений Леонтьева и Грюнайзена для кристаллических твердых тел}

В табл. 1 сопоставляются результаты расчета $\gamma$ по формуле Леонтьева (3) и уравнению Грюнайзена (2). Данные о величинах $\rho, B_{A}, v_{S}$ и $v_{L}$, входящих в формулу Леонтьева, взяты из обзора Андерсона [3]. Для рассматриваемых кристаллов ранее Беломестных и Теслевой [2] были получены значения $\gamma$ по уравнению Грюнайзена (2). Как видно, для различных кристаллов соотношение Леонтьева в целом (с некоторым разбросом данных) удовлетворительно согласуется с уравнением Грюнайзена. Здесь и далее (табл. 1 и 2 , рис. $1-4$ ) использованы данные, полученные при „стандартных“ условиях $T=298 \mathrm{~K}, p=10^{5} \mathrm{~Pa}$.

Таблица 1. Сопоставление результатов расчета параметра Грюнайзена $\gamma$ по уравнениям Грюнайзена (2) и Леонтьева (3) для кристаллических твердых тел (использованы данные [2-4])

\begin{tabular}{|c|c|c|c|c|}
\hline \multirow[b]{2}{*}{ № } & \multirow{2}{*}{$\begin{array}{c}\text { Элементы } \\
\text { и соединения }\end{array}$} & \multirow[b]{2}{*}{$\mu$} & \multicolumn{2}{|c|}{$\gamma$} \\
\hline & & & $\begin{array}{c}\text { Леонтьев } \\
\text { (3) }\end{array}$ & $\begin{array}{c}\text { Грюнайзен } \\
(2)\end{array}$ \\
\hline 1 & $\mathrm{LiF}$ & 0.214 & 1.35 & 1.34 \\
\hline 2 & $\mathrm{NaCl}$ & 0.243 & 1.53 & 1.46 \\
\hline 3 & $\mathrm{LiCl}$ & 0.245 & 1.47 & 1.52 \\
\hline 4 & $\mathrm{KCl}$ & 0.259 & 1.60 & 1.60 \\
\hline 5 & $\mathrm{NaF}$ & 0.234 & 1.44 & 1.57 \\
\hline 6 & $\mathrm{NaBr}$ & 0.270 & 1.65 & 1.56 \\
\hline 7 & $\mathrm{LiBr}$ & 0.256 & 1.53 & 1.70 \\
\hline 8 & $\mathrm{KBr}$ & 0.283 & 1.67 & 1.68 \\
\hline 9 & $\mathrm{Fe}$ & 0.292 & 1.68 & 1.68 \\
\hline 10 & KI & 0.265 & 1.6 & 1.63 \\
\hline 11 & Co & 0.357 & 1.85 & 2.10 \\
\hline 12 & $\mathrm{Al}$ & 0.340 & 2.16 & 2.11 \\
\hline 13 & $\mathrm{Ag}$ & 0.379 & 2.24 & 2.40 \\
\hline 14 & $\mathrm{Be}$ & 0.034 & 0.83 & 0.83 \\
\hline 15 & Y & 0.245 & 1.40 & 1.25 \\
\hline 11 & $\mathrm{Co}$ & 0.357 & 1.85 & 2.10 \\
\hline 12 & $\mathrm{Al}$ & 0.340 & 2.16 & 2.11 \\
\hline 13 & $\mathrm{Ag}$ & 0.379 & 2.24 & 2.40 \\
\hline 14 & $\mathrm{Be}$ & 0.034 & 0.83 & 0.83 \\
\hline 15 & Y & 0.245 & 1.40 & 1.25 \\
\hline 16 & $\mathrm{NaNO}_{3}$ & 0.257 & 1.27 & 1.31 \\
\hline 17 & $\mathrm{NaClO}_{3}$ & 0.270 & 1.61 & 1.37 \\
\hline 18 & Th & 0.254 & 1.61 & 1.40 \\
\hline 19 & $\mathrm{Mg}$ & 0.270 & 1.64 & 1.41 \\
\hline 20 & $\mathrm{RbBr}$ & 0.267 & 1.76 & 1.50 \\
\hline 21 & $\mathrm{Ta}$ & 0.337 & 2.05 & 1.73 \\
\hline 22 & $\mathrm{AgBr}$ & 0.396 & 2.62 & 2.33 \\
\hline 23 & $\mathrm{Pd}$ & 0.374 & 2.44 & 2.40 \\
\hline 24 & $\mathrm{Au}$ & 0.420 & 2.90 & 2.80 \\
\hline
\end{tabular}

Примечание. Расчет по уравнению (2) выполнен Беломестных и Теслевой [2].

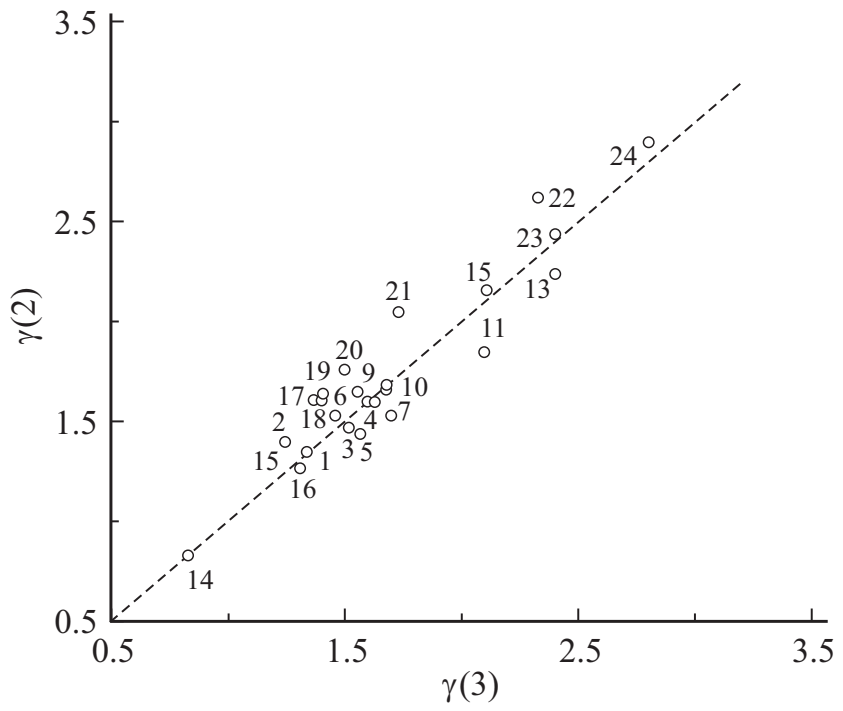

Рис. 1. Сравнение значений параметра Грюнайзена, рассчитанных по уравнению Грюнайзена $\gamma(2)$ и по формуле Леонтьева $\gamma$ (3), для различных кристаллов. Номера точек соответствуют номерам твердых тел в табл. 1.

Лучшее согласие наблюдается для квазиизотропных щелочногалоидных кристаллов с центральными силами взаимодействия частиц, что можно объяснить тем, что при выводе формулы Леонтьева помимо термодинамики, молекулярной физики, физической акустики была широко использована теория упругости, которая справедлива для изотропной сплошной среды, а заметные отклонения для ряда твердых тел (рис. 1) могут быть обусловлены анизотропией этих систем.

Причина отклонений может заключаться также в разбросе значений $\gamma$, полученных разными исследователями, а не в анизотропии тел. Так, например, по трем разным источникам параметр Грюнайзена (2) для алюминия составляет $2.11,2.43$ и 2.34 [4].

\section{3. Ангармонизм и отношение скоростей акустических волн}

Обращает внимание то обстоятельство, что в формуле Леонтьева (3) в левой части равенства находится мера ангармонизма $(\gamma)$, а в правую часть на первый взгляд входят только гармонические величины $\left(\rho, B_{A}, v_{K}^{2}\right)$. Наблюдается тем самым как бы противоречие.

На самом деле здесь мы имеем дело с кажущимся противоречием, поскольку правая часть равенства также зависит от ангармонизма за счет величины $v_{K}^{2}$, которая является функцией отношения квадратов скоростей звука $\left(v_{L}^{2} / v_{S}^{2}\right)$. В свою очередь между отношением квадратов скоростей продольной и поперечной звуковых волн и параметром Грюнайзена (2) обнаруживается линейная корреляция как для кристаллов [5], так и для стекло- 
Таблица 2. Плотность $\rho$, скорости распространения продольных $\left(v_{L}\right)$ и поперечных $\left(v_{S}\right)$ акустических волн, модуль объемного сжатия $B_{A}$ и параметр Грюнайзена $\gamma$ для стекол $\mathrm{Na}_{2} \mathrm{O}-\mathrm{Al}_{2} \mathrm{O}_{3}-\mathrm{SiO}_{2}$ (использованы данные [17])

\begin{tabular}{|c|c|c|c|c|c|c|c|c|c|}
\hline \multirow{2}{*}{ № } & \multicolumn{3}{|c|}{ Состав по синтезу, mol\% } & \multirow{2}{*}{$\begin{array}{c}\rho \cdot 10^{-3} \\
\mathrm{~kg} / \mathrm{m}^{3}\end{array}$} & \multirow{2}{*}{$\begin{array}{l}v_{L} \\
\mathrm{~m} / \mathrm{s}\end{array}$} & \multirow{2}{*}{$\begin{array}{l}v_{S} \\
\mathrm{~m} / \mathrm{s}\end{array}$} & \multirow{2}{*}{$\begin{array}{c}B_{A} \cdot 10^{-8} \\
\mathrm{~Pa}\end{array}$} & \multirow{2}{*}{$\mu$} & \multirow{2}{*}{$\gamma$} \\
\hline & $\mathrm{Na}_{2} \mathrm{O}$ & $\mathrm{Al}_{2} \mathrm{O}_{3}$ & $\mathrm{SiO}_{2}$ & & & & & & \\
\hline 1 & 15 & 0 & 85 & 2339 & 5430 & 3340 & 342 & 0.196 & 1.28 \\
\hline 2 & 15 & 5 & 80 & 2358 & 5570 & 3390 & 370 & 0.206 & 1.31 \\
\hline 3 & 15 & 10 & 75 & 2410 & 5697 & 3510 & 386 & 0.194 & 1.26 \\
\hline 4 & 15 & 15 & 70 & 2465 & 5737 & 3469 & 416 & 0.212 & 1.34 \\
\hline 5 & 15 & 20 & 65 & 2428 & 5850 & 3540 & 425 & 0.211 & 1.34 \\
\hline 6 & 15 & 25 & 60 & 2472 & 6000 & 3568 & 470 & 0.226 & 1.40 \\
\hline 7 & 25 & 0 & 75 & 2439 & 5280 & 3140 & 359 & 0.226 & 1.40 \\
\hline 8 & 25 & 5 & 70 & 2455 & 5480 & 3240 & 394 & 0.231 & 1.41 \\
\hline 9 & 25 & 10 & 65 & 2461 & 5610 & 3330 & 411 & 0.228 & 1.40 \\
\hline 10 & 25 & 20 & 55 & 2470 & 5680 & 3450 & 405 & 0.208 & 1.32 \\
\hline 11 & 25 & 25 & 50 & 2499 & 5790 & 3490 & 432 & 0.215 & 1.35 \\
\hline 12 & 25 & 30 & 45 & 2519 & 6026 & 3556 & 490 & 0.233 & 1.43 \\
\hline 13 & 35 & 0 & 65 & 2497 & 5340 & 3070 & 398 & 0.253 & 1.52 \\
\hline 14 & 30 & 5 & 65 & 2486 & 5500 & 3200 & 413 & 0.244 & 1.47 \\
\hline 15 & 20 & 15 & 65 & 2450 & 5670 & 3490 & 390 & 0.195 & 1.28 \\
\hline 16 & 17.5 & 17.5 & 65 & 2447 & 5746 & 3458 & 418 & 0.216 & 1.35 \\
\hline
\end{tabular}

образных твердых тел [6,7],

$$
\left(\frac{v_{L}}{v_{S}}\right)^{2}=A_{1} \gamma+A_{2},
$$

где $A_{1}$ и $A_{2}$ - эмпирические постоянные. В качестве примера на рис. 2 приводится линейная зависимость отношения $\left(v_{L}^{2} / v_{S}^{2}\right)$ от параметра Грюнайзена $\gamma$ для натриевоалюмосиликатных стекол.

Параметр Грюнайзена $\gamma$ оказывается однозначной функцией коэффициента Пуассона $\mu$ [см. далее соотношение (12)], а величина $\mu$ в теории упругости [8]

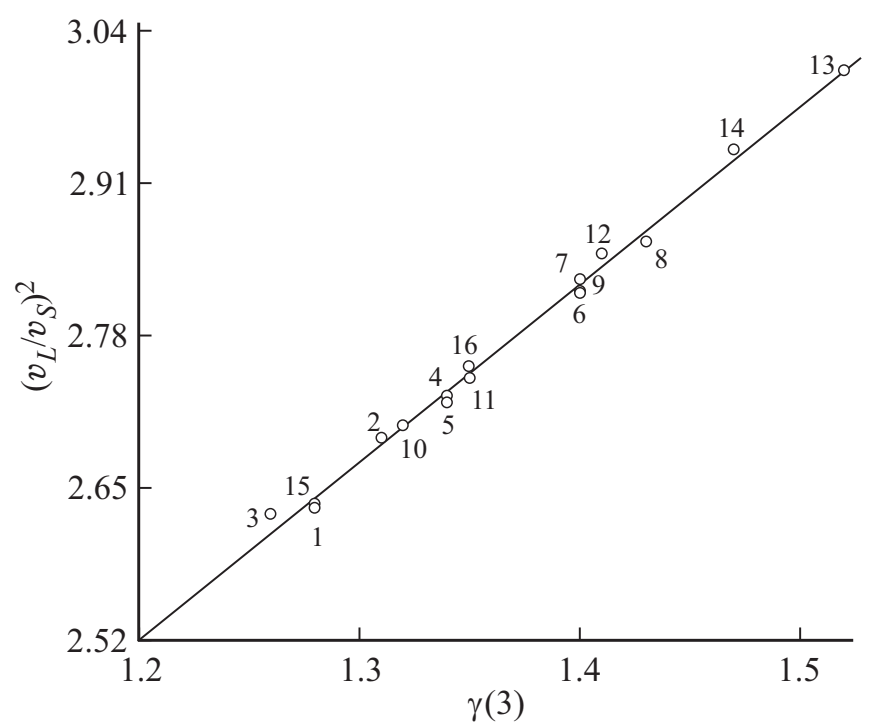

Рис. 2. Линейная корреляция между $\gamma$ и $\left(v_{L} / v_{S}\right)^{2}$ для натриевоалюмосиликатных стекол. Номера точек соответствуют номерам стекол в табл. 2. однозначно связана с отношением $\left(v_{L}^{2} / v_{S}^{2}\right)$, зависящим от ангармонизма. Взаимосвязь $\mu$ и $\gamma$ находит обоснование в теории Пинеды (Pineda) [9]. Корреляцию между гармоническими (линейными) и ангармоническими (нелинейными) характеристиками твердых тел Конторова $[10,11]$ объясняет существованием взаимосвязи гармонического $a$ и ангармонического $b$ коэффициентов в разложении потенциальной энергии взаимодействия атомов $U(r)$ в ряд Тейлора. Коэффициенты $a$ и $b$ определены соответственно второй и третьей производными функции $U(r)$ при равновесном межатомном расстоянии $r=r_{0}$. Используя в указанных производных потенциал $M u\left(U=-A r^{-m}+B r^{-n}\right)$, Конторова [10] установила следующую взаимосвязь указанных коэффициентов

$$
b=\frac{m+n+3}{2 r_{0}} a
$$

и получила функциональную зависимость от них линейных и нелинейных свойств твердых тел.

Исходя из приведенных соображений и данных, можно полагать, что в формуле Леонтьева нет противоречия, касающегося взаимосвязи гармонических и ангармонических характеристик твердых тел.

\section{4. Комментарий к выводу формулы Леонтьева}

Рассмотрим кратко приближенный вариант вывода данной формулы [1]. Из закона сохранения энергии для адиабатического процесса $(d U=d A)$

$$
C_{V} d T=p d V
$$


и из определения коэффициента объемного теплового расширения

$$
\beta=\frac{1}{V}\left(\frac{d V}{d T}\right)
$$

следует приближенное выражение

$$
p V \cong \frac{C_{V}}{\beta}
$$

Для давления используется основное уравнение молекулярно-кинетической теории идеального газа

$$
p=\frac{2}{3} \frac{\bar{U}}{V}
$$

где объемная плотность средней энергии теплового движения молекул выражается через плотность и среднеквадратичную скорость звука (4)

$$
\frac{\bar{U}}{V}=\rho v_{K}^{2}
$$

Из последних трех соотношений (5)-(7) вытекает связь тепловых характеристик $\beta$ и $C_{V}$ со скоростями распространения продольной и поперечной звуковых волн (с упругими свойствами)

$$
\frac{C_{V}}{\beta V}=\frac{2}{3} \rho v_{K}^{2}
$$

которая находится в согласии с экспериментальными данными [1]. Установление связи между упругими и тепловыми свойствами веществ является основной задачей работы Леонтьева [1]. Формула (3) для параметра Грюнайзена применялась при решении данной задачи.

С привлечением равенства (8) из уравнения Грюнайзена (2) в приближении $B \approx B_{A}$ выводится формула Леонтьева (3)

$$
\gamma=\frac{\beta B V}{C_{V}}=\left(\frac{\beta V}{C_{V}}\right) B=\frac{3}{2}\left(\frac{B}{\rho v_{K}^{2}}\right) \cong \frac{3}{2}\left(\frac{B_{A}}{\rho v_{K}^{2}}\right) .
$$

Приближение $B \approx B_{A}$ оправдано из-за малости коэффициента теплового расширения твердых тел $\beta \approx 10^{-4}-10^{-5} 1 /$ град $\left(B_{A} / B=1+\gamma \beta T\right)$. Тем не менее необходимо сделать оговорку, что это приближение справедливо при низких температурах вдали от температуры плавления.

Наряду с приближенным вариантом Леонтьев дает строгий вывод формулы (3) без использования уравнения Грюнайзена (2) [1]. Однако и в строгом варианте вывода также встречается основное уравнение молекулярно-кинетической теории идеального газа (6).

В связи с этим, возникает вопрос об оправданности применения уравнения (6) к твердым телам.

Один из вероятных ответов на данный вопрос заключается в том, что Леонтьев под давлением $p$ в выражении (6), по-видимому, подразумевает фононное давление [12,13], поскольку в работе [1] он нигде не употребляет термины типа „давление идеального газа“, „уравнение кинетической теории газа“. Не используется также, впрочем, термин „фононное давление“. Формула (6) записывается им после предложения: „Затем применим следующее соотношение классической и квантовой физики для связи давления со средней энергией молекулы“ [1], причем без ссылок на какие-либо источники. Известно, что формула для фононного давления в твердом теле подобна формуле давления газа (6).

Другой вариант ответа на поставленный выше вопрос можно получить из представления Френкеля $[13,14]$ о возможности применения формулы (6) к твердым телам. Ниже в кавычках взяты выражения (предложения), заимствованные из книг Френкеля $[13,14]$.

Френкель [13, с. 169] отмечает, что „тепловое давление, которое оказывает атом, связанный почти упругим образом в твердом теле, на один из боковых атомов, по своей зависимости от температуры очень похоже на то давление, которое он оказывал бы, будучи совершенно свободным, т.е. если бы мы имели дело не с моделью твердого тела, а с моделью газа“. Он предлагает вывод формулы для коэффициента теплового расширения твердого тела, основанный на применении формулы давления идеального газа (6) $[13,14]$.

Причиной теплового расширения по Френкелю с формальной точки зрения является давление

$$
p=B \frac{\Delta V}{V}
$$

„численно равное тому внешнему давлению (отрицательного знака), которое могло бы вызвать то же самое изменение объема $\Delta V$ при $T=0 \mathrm{~K}$, но приложенное к поверхности тела с внутренней ее стороны“ (см. [14], гл. XVI, §59). По своей природе это давление $p$ не имеет ничего общего с упругим напряжением, которое им вызывается и уравновешивается. „Будучи непосредственно обусловленным тепловым движением, оно представляет собой не что иное, как равнодействующую сил, испытываемых. . . внутренними атомами со стороны поверхностных, которые стремятся от них оторваться“.

„Если бы взаимная связь атомов внезапно исчезла, то для удержания их в данном объеме $V$ пришлось бы приложить внешнее давление $p$, которое определяется известной формулой кинетической теории газов“

$$
p V=\frac{2}{3} \bar{U}
$$

„Поскольку $p$, определяемое (6), может на самом деле компенсировать силы межатомного сцепления, оно должно совпадать с введенным выше давлением $p^{66}(9)$ [14].

Таким образом, с этих позиций использование основного уравнения молекулярно-кинетической теории идеального газа (6) в работе Леонтьева вполне оправдано. 


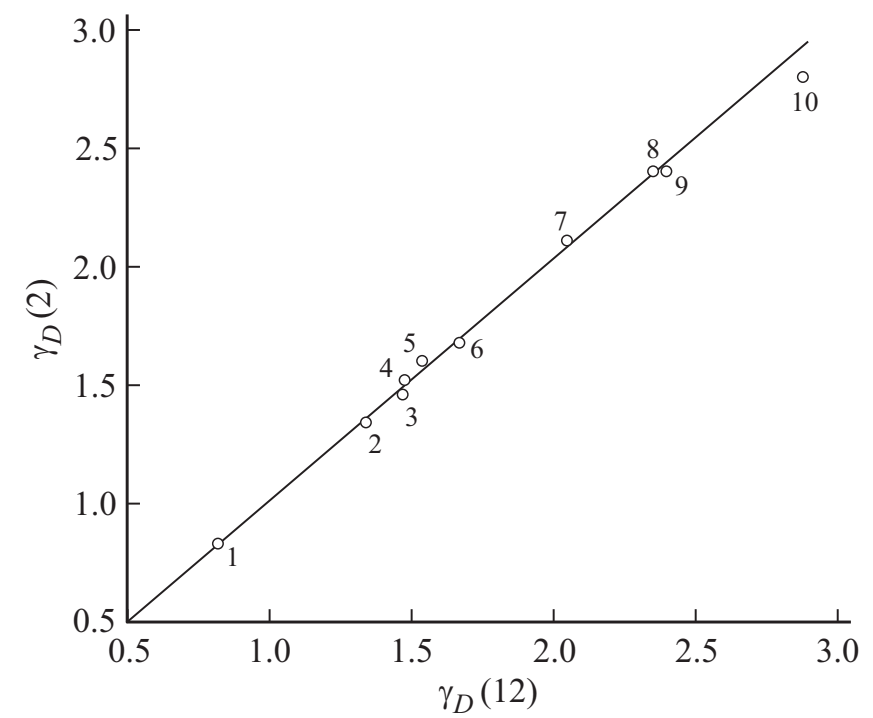

Pис. 3. Линейная корреляция между значениями параметра Грюнайзена $\gamma_{D}$, полученными по уравнению Грюнайзена $\gamma_{D}(2)$ и по формуле Беломестных-Теслевой $\gamma_{D}(12)$, для различных твердых тел (использованы данные [2,15]). $1-\mathrm{Be}, 2-\mathrm{LiF}$, $3-\mathrm{NaCl}, 4-\mathrm{LiCl}, 5-\mathrm{KCl}, 6-\mathrm{KBr}, 7-\mathrm{Al}, 8-\mathrm{Ag}$, $9-\mathrm{Pd}, 10-\mathrm{Au}$.

\section{5. Вывод формулы Беломестных-Теслевой из уравнения Леонтьева}

Используя выражение для модуля сдвига $G=\rho v_{S}^{2}$ и формулу (4) для квадрата среднеквадратичной скорости звука $v_{K}^{2}$, преобразуем уравнение Леонтьева (3), исключив $v_{K}^{2}$,

$$
\gamma=\frac{3}{2}\left(\frac{B_{A}}{\rho v_{S}^{2}}\right) \frac{v_{S}^{2}}{v_{K}^{2}}=\frac{3}{2}\left(\frac{B_{A}}{G}\right) \frac{3}{\left(v_{L} / v_{S}\right)^{2}+2} .
$$

Далее с помощью известных соотношений теории упругости [8]:

$$
\frac{B}{G}=\frac{2}{3} \frac{1+\mu}{1-2 \mu}, \quad\left(\frac{v_{L}}{v_{S}}\right)^{2}=\frac{2-2 \mu}{1-2 \mu},
$$

где $\mu-$ коэффициент Пуассона, в приближении $B_{A} \approx B$ приходим к формуле Беломестных-Теслевой [2]:

$$
\gamma=\frac{2}{3}\left(\frac{1+\mu}{1-2 \mu}\right)
$$

которая была получена авторами [2] из иных исходных посылок без привлечения уравнения Леонтьева (3), причем достаточно строго на основе теории упругости, молекулярной акустики и термодинамики. Авторы назвали $\gamma$ упругим параметром Грюнайзена.

Примечательно то обстоятельство, что такая сравнительно простая формула (12) позволяет рассчитывать $\gamma$ по данным только о коэффициенте Пуассона $\mu$.
Расчет параметра Грюнайзена с помощью формулы Беломестных-Теслевой для металлов, ионных и молекулярных кристаллов удовлетворительно согласуется с результатами расчета по уравнению Грюнайзена (2) $[2,15,16]$ (рис. 3$)$.

\section{6. Применение формулы Леонтьева к стеклам}

По формуле Леонтьева (3) нами рассчитан параметр Грюнайзена различных стекол, у которых известны данные о величинах $\rho, v_{S}, v_{L}$ и $B_{A}[17-23]$. У большинства исследованных стекол полученные значения $\gamma \approx 1-2$ близки к данным для квазиизотропных ионных кубических кристаллов. В качестве примера в табл. 2 приводятся значения $\gamma$, полученные с помощью уравнения Леонтьева (3) для натриевоалюмосиликатных стекол.

В щелочносиликатных стеклах [17] наблюдается закономерное усиление ангармонизма колебаний решетки с ростом содержания ионов щелочных металлов. Так, например, в стеклах $\mathrm{Na}_{2} \mathrm{O}-\mathrm{SiO}_{2}$ при содержании $\mathrm{Na}_{2} \mathrm{O}: 15,25$ и $35 \mathrm{~mol} \%$ значения $\gamma$ равны 1.28, 1.40 и 1.52 (табл. 2). Параметр Грюнайзена кварцевого стекла $\mathrm{SiO}_{2}$ составляет 1.15 . У стеклообразных метафосфатов щелочноземельных металлов $R \mathrm{O}-\mathrm{P}_{2} \mathrm{O}_{5} \quad(R=\mathrm{Mg}, \mathrm{Ca}, \mathrm{Sr}, \mathrm{Ba}) \quad[20]$ при содержании $50 \mathrm{~mol} \% \quad R \mathrm{O}$ значение $\gamma$ растет в ряду $\operatorname{Mg}(\gamma=1.2)-\mathrm{Ca}(\gamma=1.57)-\mathrm{Sr}(\gamma=1.62)-\mathrm{Ba}(\gamma=2.1)$. $\mathrm{y}$ стеклообразных халькогенидов $\mathrm{As}-\mathrm{Se}$ и As-S [21, c. 176] значения $\gamma$ сравнительно малы $\gamma=1.1-1.25$. Стеклообразный селен $\mathrm{Se}$ имеет преимущественно цепочечную структуру. Введение мышьяка приводит к сшиванию части цепей. Поэтому при переходе от Se к стеклу As-Se величина $\gamma$ падает (с 1.32 до 1.15 у $\mathrm{As}_{2} \mathrm{Se}_{3}$ ). У силикатных стекол $\mathrm{Na}_{2} \mathrm{O}-\mathrm{Al}_{2} \mathrm{O}_{3}-\mathrm{SiO}_{2} \quad[17], \quad \mathrm{Na}_{2} \mathrm{O}-\mathrm{Li}_{2} \mathrm{O}-\mathrm{SiO}_{2} \quad[18]$ и $\mathrm{Li}_{2} \mathrm{O}-\mathrm{Al}_{2} \mathrm{O}_{3}-\mathrm{SiO}_{2}[19]$ значения $\gamma$ меняются в пределах 1.2-1.5. Из табл. 2 видно, что величина $\gamma$ при изменении содержания окислов в натриевоалюмосиликатных стеклах меняется вполне определенным образом (хотя и в небольших пределах). Например, при росте содержания $\mathrm{Al}_{2} \mathrm{O}_{3}$ с 0 до $25 \mathrm{~mol} \%$ значение $\gamma$ растет с 1.28 до 1.40 (при $15 \mathrm{~mol} \% \mathrm{Na}_{2} \mathrm{O}$ ).

Таким образом, расчет параметра Грюнайзена стеклообразных систем по формуле Леонтьева (3) приводит к вполне разумным значениям $\gamma$.

Для стекол не так просто найти необходимые экспериментальные данные о величинах, входящих в уравнение Грюнайзена (2), особенно данные о тепловых характеристиках. Поэтому для исследованных стекол результаты расчета по формуле Леонтьева сравнили со значениями $\gamma$, следующими из уравнения Беломестных-Теслевой (12).

На рис. 4 приводится зависимость параметра Грюнайзена $\gamma$, рассчитанного по формуле Леонтьева, от функции коэффициента Пуассона по Беломестных-Теслевой 


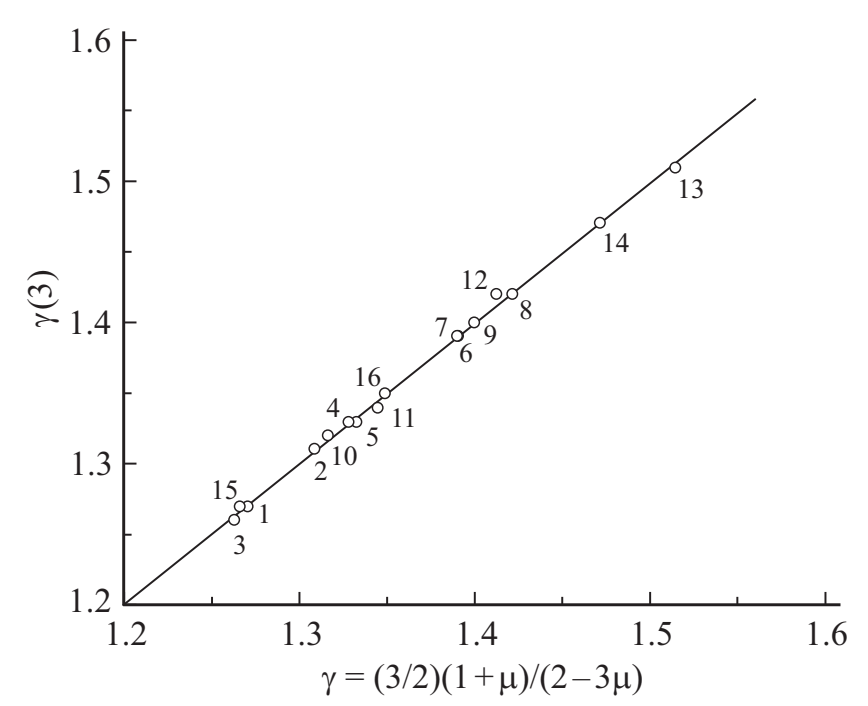

Рис. 4. Сравнение значений параметра Грюнайзена, рассчитанных по уравнению Леонтьева $\gamma$ (3) и по формуле Беломестных-Теслевой (12), для натриевоалюмосиликатных стекол. Номера точек соответствуют номерам стекол в табл. 2.

$(3 / 2)(1+\mu) /(2-3 \mu) \quad$ для $\quad$ натриевоалюмосиликатных стекол с различным содержанием окислов (табл. 2). Видно, что точки ложатся на прямую с тангенсом угла наклона, равным единице, что указывает на хорошее согласие между уравнениями Леонтьева и Беломестных-Теслевой. Такие же результаты получены для других исследованных стекол.

\section{7. Заключение}

В отличие от известного соотношения Грюнайзена, полученного из уравнения состояния, формула Леонтьева выведена непосредственно из определения параметра Грюнайзена путем усреднения частоты нормальных мод колебаний решетки. Она привлекательна тем, что позволяет рассчитывать $\gamma$ по более доступным экспериментальным данным. Установлено, что для металлов, ионных и молекулярных кристаллов формула Леонтьева находится в удовлетворительном согласии с уравнением Грюнайзена. С помощью формулы Леонтьева проведен расчет параметра Грюнайзена различных стеклообразных твердых тел (щелочносиликатные, натриевоалюмосиликатные стекла, стеклообразные халькогениды, метафосфаты щелочноземельных металлов). Получились разумные результаты. Показано, что из уравнения Леонтьева можно вывести формулу Беломестных-Теслевой, которая дает возможность рассчитывать параметр Грюнайзена по экспериментальным данным только коэффициента Пуассона. Однозначная связь параметра Грюнайзена с коэффициентом Пуассона означает, что ангармонизм $(\gamma)$ определяет механизм относительной поперечной деформации $(\mu)$, что в дальнейшем требует детального исследо- вания. Уравнения Леонтьева и Беломестных-Теслевой несомненно заслуживают внимания. Применительно к стеклам они находятся в хорошем согласии между собой.

\section{Благодарности}

Выражаем благодарность профессору В.Н. Беломестных за ценные замечания, высказанные при обсуждении работы.

\section{Финансирование работы}

Работа выполнена при финансовой поддержке Министерства науки и высшего образования РФ (грант № 3.5406. 2017/Б4).

\section{Конфлликт интересов}

Авторы заявляют, что у нас нет конфликта интересов.

\section{Список литературы}

[1] К.Л. Леонтьев. Акуст. журн. 27, 554 (1981).

[2] В.Н. Беломестных, Е.П. Теслева. ЖТФ 74, 140 (2004).

[3] O. Anderson. In: Physical Acoustics. Vol. III. Part B. Lattice Dynamics / Ed. W.P Mason. Academic, N.Y.-London (1965). $62-121 \mathrm{p}$.

[4] В.Н. Беломестных. Письма в ЖТФ 30, 14 (2004).

[5] Д.С. Сандитов, А.А. Машанов, М.В. Дармаев. ЖТФ 79, 155 (2009).

[6] Д.С. Сандитов, А.А. Машанов, Б.Д. Сандитов, С.Ш. Сангадиев. ЖТФ 81, 44 (2011).

[7] Д.С. Сандитов, С.Б. Мункуева, Д.З. Батлаев, Б.Д. Сандитов. ФТТ 54, 1540 (2012).

[8] Л.Д. Ландау, Е.М. Лифшиц. Теория упругости. 3-е изд. Наука, М. (1965). 204 c.

[9] E. Pineda. Phys. Rev. B73, 104109 (2006).

[10] Т.А. Конторова. В кн.: Некоторые проблемы прочности твердых тел. Изд-во АН СССР, М.-Л. (1959). С. 99-107.

[11] В.П. Жузе, Т.А. Конторова. ЖТФ 28, 727 (1958).

[12] А.И. Бурштейн. Молекулярная физика. Наука, Новосибирск (1986). $285 \mathrm{c}$.

[13] Я.И. Френкель. Введение в теорию металлов. Гостехиздат, Л.-М. (1948). $291 \mathrm{c}$.

[14] Я.И. Френкель. Электрическая теория твердых тел. Изд-во М. и С. Сабашниковых, СПб. (1924). 183 с.

[15] Д.С. Сандитов, В.Н. Беломестных. ЖТФ 81, 77 (2011).

[16] Д.С. Сандитов, В.В. Мантатов, Б.Д. Сандитов. ФТТ 51, 947 (2009).

[17] В.Я. Лившиц, Д.Г. Теннисон, С.Б. Гукасян, А.К. Костанян. Физ. и хим. стекла 8, 688 (1982).

[18] Г.О. Карапетян, В.Я. Лившиц, Д.Г. Теннисон. Физ. и хим. стекла 7, 188 (1981). 
[19] Г.О. Карапетян, В.Я. Лившиц, Д.Г. Теннисон. Физ. и хим. стекла 5, 314 (1979).

[20] Е.А. Гурович, А.А. Ильин, А.А. Пронкин. Физ. и хим. стекла 5, 383 (1979).

[21] В.В. Тарасов. Проблемы физики стекла. Стройиздат, М. (1979). $256 \mathrm{c}$.

[22] О.В. Мазурин, М.В. Стрельцина, Т.Н. Швайко-Швайковская. Свойства стекол и стеклообразующих расплавов. Справочник. Т. 1-6. Наука, Л.-СПб (1973-1998).

[23] MDL ( SciGlass-7.8. Institute of Theoretical Chemistry, Shrewbury, MA, (2012). www.sciglass.info

Редактор Ю.Э. Китаев 